\title{
Multispectral gas detection method
}

\author{
M. Kastek, T. Sosnowski, T. Orżanowski, K. Kopczyński \\ \& M. Kwaśny \\ Institute of Optoelectronics, Military University of Technology, Poland
}

\begin{abstract}
The article presents the problem of methane detection with a multispectral infrared camera. It also presents some commercially available devices and method used for the detection of chemical substances (gases). The project of a multispectral infrared camera and theoretical calculation regarding the possibility of methane detection is also reported in this paper. The calculations included the properties of optical path: camera-cloud of methane background. Verification of theoretical results was made during laboratory measurement. Some initial results of methane detection are also presented.
\end{abstract}

Keywords: gas detection, passive detection of gases, multispectral detection.

\section{Introduction}

Thermal cameras (fitted with a set of appropriate filters and special software) and IR imaging spectroradiometers make it possible to obtain the image of observed scenery with marked areas, where specific gaseous chemical compounds can be found. The detection of gases is possible, because absorption and emission bands of many chemical compounds (molecular vapours and aerosols) are located in the IR range. Two spectral bands are usually analysed: $3-5 \mu \mathrm{m}$ and $8-12 \mu \mathrm{m}$, which are the typical working bands of IR devices due to atmospheric transmission. The development of imaging devices for gas detection was greatly influenced by the progress in focal plane array IR detectors. The modern devices fitted with highly sensitive IR detectors can detect the presence of chemical compounds from the distance of $1-2$ kilometres therefore they must have high temperature and spectral resolution. Two main groups of such devices can be distinguished: thermal cameras (additionally equipped with spectral filters and signal processing units) and devices built around IR spectroradiometers. 
Gas detection by thermal cameras consists in the comparison of two thermal images: one taken in the absorption (or emission) band of sought after gas and the other (so called reference image) taken slightly outside spectral area where given gas exhibits absorptive (or emissive) properties.

\section{Methods and devices used for the detection of gases}

Thermal camera used for detection of volatile chemical substances has to be equipped with the systems ensuring adequate spectral resolution. It is connected with the necessity of application of the filters (usually tunable ones, increasing universality of a device), the systems of signal analysis, and often special optical system.

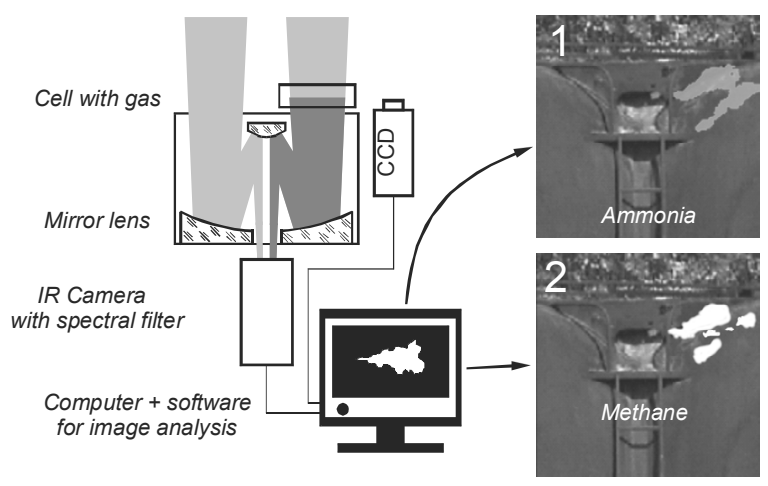

Figure 1. Gas detection system with a thermal camera [1].

Sample application of a thermal camera equipped with an optical filter for gas detection is schematically presented in Fig. 1. The system consists of: an Agema THV900LW camera equipped with the bandpass filters having the characteristics matched to the absorption bands of gases, a Cassegrainian telescope, frame grabber, CCD camera, and PC computer with special software for image analysis. The IR camera is equipped with suitable interference filters to isolate narrow spectral region containing absorption features of the gas. The image processing is performed according to the following scheme: $\mathrm{A}$ and $\mathrm{B}$ images are captured at the same time using the IR camera and frame grabber (A - image of the infrared scene from one of telescope openings, B - image of the same scene from the other telescope opening, with gas cell in front of it), next an error normalization image $\mathrm{E}$ is created to handle imperfections such as symmetrical vignette and stray-light from the two openings $\left(E=A_{0} / B_{0}\right.$ where $A_{0}, B_{0}$ are images registered with no gas in the scene). Finally images are digitally overlapped and gas correlation image $G$ is calculated as $G=A / B / E$. In this way the appropriate offset is subtracted from the images. Resulting image, which presents the region of gas presence in the observed scenery, is superposed on a visual image from CCD camera [1]. The result of these operations is shown in Fig. 1. Image (1) presents the total leakage of ammonia. Due to filling up the gas 
chamber with methane, detection of this substance and its visualization is also possible - image (2).

Another approach of a thermal camera application for the searching of gas leakages is a thermal camera system equipped additionally with a Fabry-Perot interferometer. An interferometer in IR system plays the role of a tunable optical filter (Fig. 2). It selects wavelength of IR radiation illuminating, at the given moment, the pixels of FPA of a thermal camera. An operation range of the interferometer is $3-5 \mu \mathrm{m}$ (MWIR version) or $8-12 \mu \mathrm{m}$ (LWIR) and tuning velocity is of about $10-20 \mathrm{~ms}$. An interferometer module, containing a detector matrix, has been developed by the Physical Sciences, Inc. and it is known as AIRIS (Adaptive InfraRed Imaging Spectroradiometer) [2].

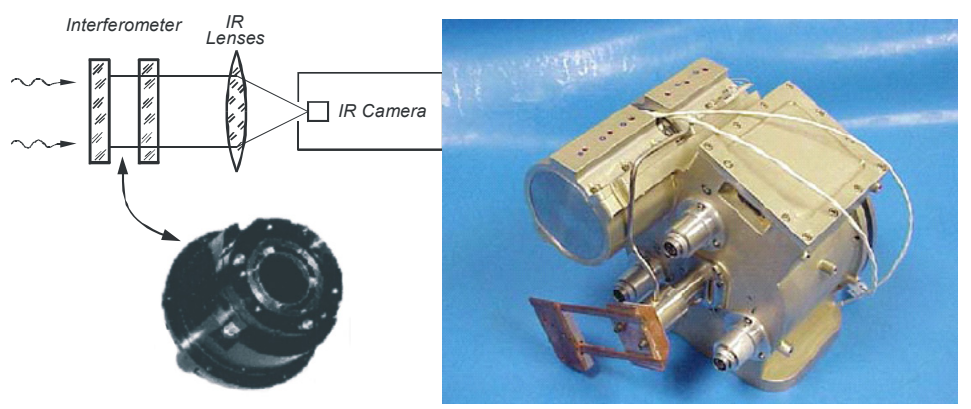

Figure 2. The scheme of thermal camera and Fabry-Perot interferometer [2].

By applying the aforementioned tunable optical filters it is possible to create a gas detection system around a thermal camera. The filter is tuned to the absorption band of a chosen compound and the interpretation of thermal image is performed by a human operator. The gas cloud appears as a "black smoke" on the display of a thermal camera. It should be mentioned that only detection of certain gas can be achieved without the possibility of quantitative analysis [2].

The Telops has developed an innovative instrument that can not only provide an early warning for chemical agents and toxic chemicals, but also one that provides a "Chemical Map" of the field of view. To provide to best field imaging spectroscopy instrument, Telops has developed the FIRST, Field-portable Imaging Radiometric Spectrometer Technology (Fig. 3). This instrument is based on a modular design that includes: a high performance infrared FPA and data acquisition electronics, onboard data processing electronics, a high performance Fourier transform modulator, dual integrated radiometric calibration targets and a visible boresighted camera. These modules, assembled together in an environmentally robust structure, used in combination with Telops' proven radiometric and spectral calibration algorithms make this instrument a worldclass passive standoff detection system for chemical imaging [3].

There are several systems and devices for gas detection in the infrared range that use thermal camera or IR-sensitive FPA, like GasFindIR produced by FLIR Systems. The analysis showing the estimated gas detection capabilities as 




Figure 3. The Field-portable Imaging Radiometric Spectrometer Technology FIRST [3].

a function of gas concentration and NETD of a thermal camera will be presented in the following part of this paper.

\section{Thermal camera capability for gas detection}

The computer simulation of a methane gas detection process was performed in order to estimate the detection capabilities of a thermal camera. The variation of a signal reaching the camera caused by the presence of a gas was calculated and compared with the reference signal obtained without the presence of a gas in the camera's field of view.

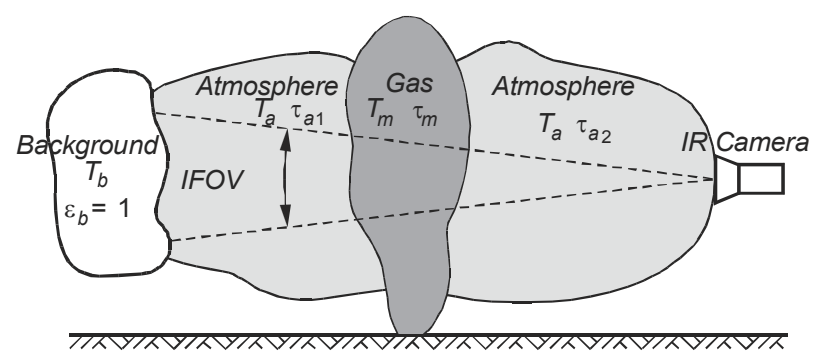

Figure 4. Scheme of a measuring process and notations accepted for gas analysis in atmosphere.

When there is a methane gas in a field of view of the thermal camera (fig. 4), total infrared radiance reaching the sensor at given wavelength $N_{M S C}$ is the sum of the contributions from each layer and given by:

$$
N_{M S C}(\lambda)=\tau_{M S C}\left[\tau_{a} \tau_{m} N_{b}\left(\lambda, T_{b}\right)+\tau_{a}\left(1-\tau_{m}\right) N_{m}\left(\lambda, T_{m}\right)+\left(1-\tau_{a}\right) N_{a}\left(\lambda, T_{a}\right)\right] .
$$

where $N_{b}$ is the Planck radiance of the background, $N_{m}$ is radiance of the methane cloud and $N_{a}$ is the atmospheric radiance. The quantities $\tau_{m}$ and $\tau_{a}$ are the spectral transmission of the methane cloud and the transmission of the atmosphere between the methane cloud and IR camera. The $\tau_{M S C}$ represents total spectral transmission of the objective, spectral filters and detector window. 
The radiance from the background, attenuated by the gas cloud and intervening atmosphere, is the first term in (1). The second term is the radiance of the methane cloud, also attenuated by the atmosphere between the cloud and the camera. The third term in (1) is the radiance of the atmosphere between the gas cloud and the camera.

The transmission of the methane cloud $\tau_{m}$ is computed from the spectral properties of the chemical species using Beers' Law:

$$
\tau_{m}(\lambda)=\exp \left[-\sum k_{i}(\lambda) C_{i} d\right]
$$

where $C_{i}$ is the average concentration of the chemical compound over the optical path length $\mathrm{d}$ and $k_{i}(\lambda)$ is the wavelength-dependent absorption coefficient [2]. The sum over index $i$ in (2) is calculated over all spectrally relevant chemical species.

PC MODWIN 3 v.1.0 computer program was used for the calculation of the transmission of the methane cloud. The results of this simulation are presented in Fig. 5.

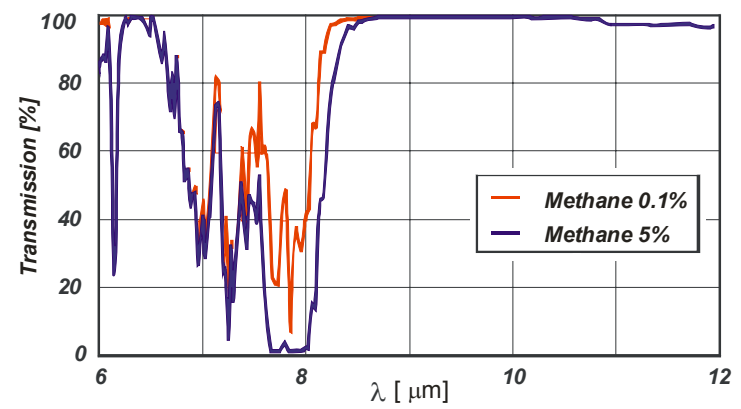

Figure 5. The transmission of the methane cloud for various gas concentrations.

The differential radiance observed by the camera, resulting from the presence of the gas cloud can be approximated as:

$$
\Delta N_{M S C}(\lambda)=k_{i}(\lambda) \Psi\left[\frac{d N}{d T}\right] \Delta T,
$$

where $\Delta T$ is the temperature difference between background and methane cloud $\left(T_{m}-T_{b}\right)$, and $\Psi$ is the column density of the methane.

This approach has been commonly applied to the analysis of detection of chemical agents and their simulants by all passive sensors.

NETD is s an important figure of merit used to characterize the performance of thermal imaging systems. Some analyses were performed of the NETD values required for a successful detection of a gas cloud. The relation between camera NETD and temperature difference between background $\left(T_{b}=293 \mathrm{~K}\right)$ and gas cloud that allows for gas detection (with gas concentration as a parameter) is presented in Fig. 6 [4]. 


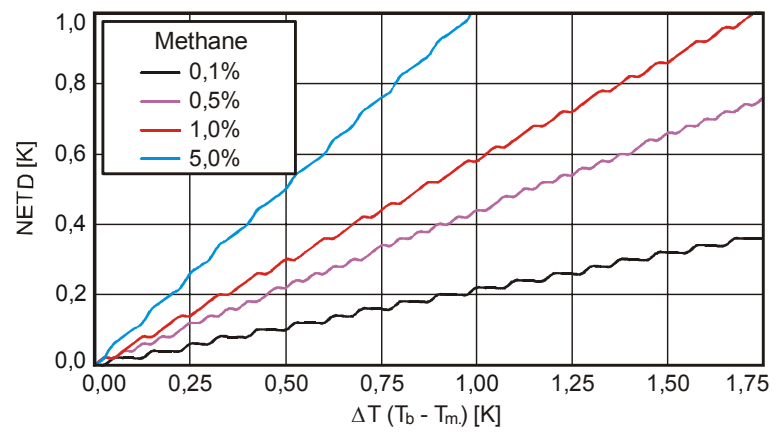

Figure 6. Required camera NEDT as a function of temperatures difference between background (object) and gas for various values of methane concentration.

It can be stated, on the basis of the presented analyses, that for typical measuring tasks it is possible to detect chemical compounds using a camera with NETD lower than $0.2 \mathrm{~K}$. Thermal detection of chemical substances is more and more frequently used method for the monitoring of industrial installations. Having in view a serious progress in IR array detectors, it should be supposed that such solutions will be commonly applied.

\section{Project of multispectral IR camera}

A multispectral IR camera, the scheme of which is shown in Fig. 7, consists of the following basic blocks: objective, set of optical filters, microbolometer FPA, electronic read-out system and image analysis system.

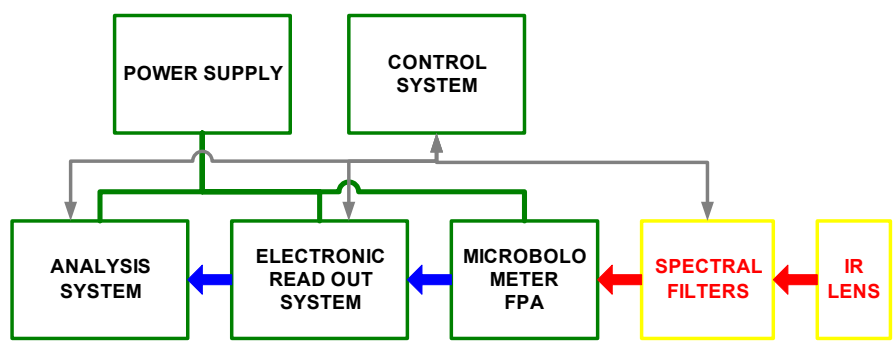

Figure 7. Block diagram of multispectral IR camera.

The Umicore Gasir Standard Lens $60 \mathrm{~mm} \mathrm{F/1.1} \mathrm{was} \mathrm{used} \mathrm{as} \mathrm{the} \mathrm{objective} \mathrm{of}$ the presented multispectral IR camera. It has the field of view of $18.5^{\circ}$ and the transmission better than 92\% in LWIR range. The UL 03081 (ULIS, France) microbolometer array detector working in the long wavelength infrared (LWIR) range of $8-14 \mu \mathrm{m}$ was used as a detector of the camera. It consists of $384 \times 288$ microbolometers (pixels) with the $35 \mu \mathrm{m}$ pixel pitch. The chosen FPA is made in the photolithographic technology based on amorphous silicon and it features 
high performance: responsivity of $7.2 \mathrm{mV} / \mathrm{K}$, thermal time constant less than $7 \mathrm{~ms}$, and NETD equal to $40 \mathrm{mK}$ at the $\mathrm{F} / 1$ optical aperture, 303K FPA temperature, and $60 \mathrm{~Hz}$ frame rate.

For the tests, a laboratory circuit for signal readout from the FPA has been designed, as well as a complete path of digital processing, data collecting, and image displaying on VGA monitor. A clock signals generator to control the readout integrated circuit (ROIC) in the FPA and all modules for digital signal processing have been made by means of Altera DSP Development Kit Stratix II Edition with the field programmable gate array (FPGA) EP2S60-1020C4 device. The raw image data were received from a FPA board with the 14-bit resolution [5]. The whole system that analyses the image from a microbolometric FPA has been designed in a FPGA chip [6]. This system was also used to control the optical filters between the detectors array and objective lens. A set of optical filters consists of two filters: methane filter 1 and methane filter 2, the transmission band of both filters covers the methane absorption area in a long IR wavelength range. These filters have been purchased from Spectrogon. Methane filter 1 consists of two filters: SP-8855 and LP-6500 and methane filter 2 consists of the SP-8100 filter and LP-6500 filter. As a reference filter, the BP 8350-12660 filter was used, also made by Spectrogon. Its transmission band is, in turn, outside the area of methane absorption. Transmission characteristics of those filters are shown in Fig. 8. The characteristics have been measured by means of Backman FAR IR 720 spectrophotometer.

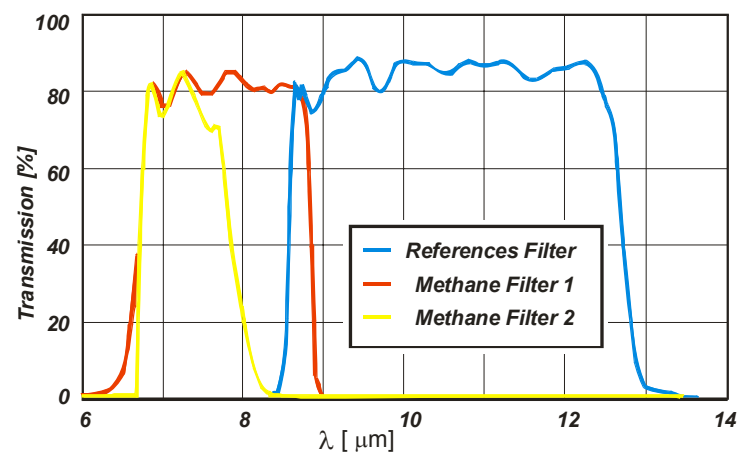

Figure 8. The transmission of optical filters used in multispectral IR camera.

The model of radiation transmission has been developed to calculate the amount of radiation reaching the input aperture of the optical system and camera's detector. The calculations were performed using PC MODWIN 3 v.1.0 software. A horizontal transmission path was assumed and Midlatitude Summer atmosphere model was chosen, for which the spectral characteristics of the atmosphere visibility coefficient $\tau_{a}$ have been determined. A series of additional calculations of a transmission coefficient for various parameters have been made to obtain a complete analysis of the influence of an atmosphere transmission coefficient on the possibility of gas detection. 
The spectral characteristic of the objective and optical filters used in the multispectral IR camera can be described by the following relation:

$$
\tau_{M S C}=\tau_{o} \tau_{F},
$$

where $\tau_{o}$ is the spectral characteristic of the objective and $\tau_{F}$ is the spectral characteristic of the actually used filter.

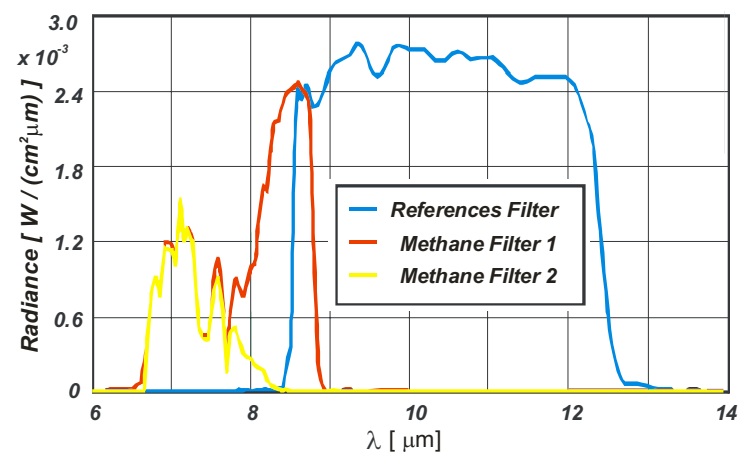

Figure 9. Spectral distribution of radiance reaching the detector of multispectral IR camera (background temperature $T_{b}=293 \mathrm{~K}$, atmosphere temperature $T_{a}=295 \mathrm{~K}$, methane concentration $5 \%$ ).

On the basis of (1) and spectral characteristic of the camera detection path described by (4), the spectral distribution of luminance causing the output electric signal of the detector during methane cloud detection can be calculated. Assuming the background temperature $T_{b}=293 \mathrm{~K}$ and the atmosphere temperature $T_{a}=295 \mathrm{~K}$, the spectral distribution of radiance has been calculated and the results are presented in Fig. 9 [7].

Spectral distribution of luminance causing the output electric signal of the detector (when reference filter was used) can be calculated according to the relation:

$$
N_{B G}(\lambda)=\tau_{M S C} \tau_{a} N_{b}\left(\lambda, T_{b}\right)
$$

The simulations were carried out for several methane concentrations and various temperatures of a background and a gas cloud to determine the possibility of gas cloud detection. To estimate the possibility of methane cloud detection using the multispectral camera, the analysis of the signal ratio, expressed by (6), was made.

$$
S=\frac{N_{B G}(\lambda)}{N_{M S C}(\lambda)}
$$

On the basis of the results of computer simulations, it can be stated that the multispectral IR camera with aforementioned parameters will provide detection 
of methane if its concentration is above 5\% and for temperature difference between the background and atmosphere of, at least, $2.3 \mathrm{~K}$. The measurements on laboratory stand have been performed to verify theoretical results.

\section{Laboratory tests of multispectral IR camera}

In order to verify theoretical calculations, laboratory tests of the designed multispectral IR camera have been performed. Figure 10 illustrates the model of multispectral IR camera used during the tests.

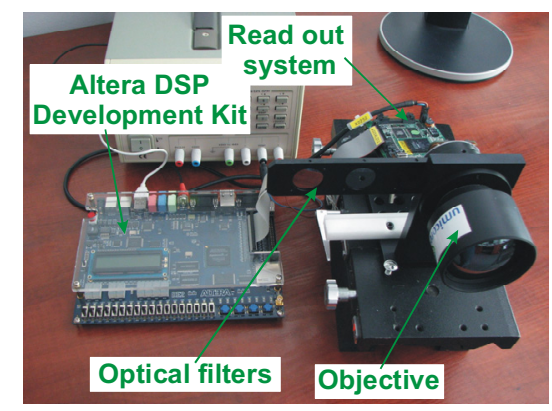

Figure 10. The model of multispectral IR camera.

The measurements were basically made to determine the possibility of methane detection. For two various methane concentrations (5\% and 1\%) a series of thermal images were registered, as presented in Fig. 11. During the measurements, the temperature difference between methane and background $\left(T_{m}-T_{b}\right)$ was $2.6 \mathrm{~K}$. To distinguish an area of methane leakage, the first thermogram was registered with the reference filter applied. This was the reference image. The following images were recorded with the appropriate methane filters. Next, due to digital image processing, the data obtained inside and outside methane absorption range were superimposed and clearly visible methane cloud has been obtained.
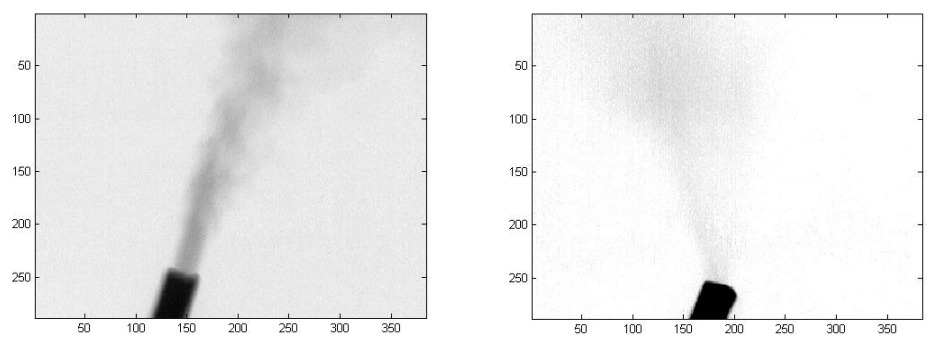

Figure 11. The thermograms registered during the tests: a) thermogram with methane concentrations $5 \%$, b) thermogram with methane concentrations $1 \%$. 
Summing up, it can be stated that a thermal camera equipped with the adequately chosen set of optical filters and the software for image processing can be used as an imaging detector for gas detection.

During theoretical analysis and laboratory verification it was proven that the microbolometer FPA has sufficient sensitivity to be applied for methane detection. It can reduce the costs of devices used for methane detection and enable real-time observations of gas leakages.

\section{References}

[1] Sandsten J., Weibring P., Edner H. and Svanberg S., Real-time gascorrelation imaging employing thermal background radiation, OPTICS EXPRESS 92, vol. 6, No. 4, (2000).

[2] Gittins M., Marinelli W. J. and Jensen J. O., Remote Sensing and Selective Detection of Chemical Vapor Plumes by LWIR Imaging Fabry-Perot Spectrometry, Proc. SPIE 4574, pp. 63-71, (2001).

[3] Vallières A., Chamberland M., Farley V., Belhumeur L., Villemaire A., Giroux J. and Legault J. F., High-performance field-portable imaging radiometric spectrometer technology for chemical agent detection, SPIE 5590, (2005).

[4] Kastek M., Madura H., Sosnowski T. and Firmanty K., Thermovision method of gas detection in far infrared range, Proc. of the 9 Advanced Infrared Technology and Applications 2007, Leon Mexico, pp. 387-394, (2008).

[5] Orżanowski T., Madura H., Powiada E. and Pasierbiński J., Analysis of a readout circuit for microbolometer detectors array, Measurements, Automation, and Checking, No. 9, pp. 16-20, Poland, (2006).

[6] Sosnowski, T., Orżanowski, T., Kastek, M., Chmielewski, K., Digital image processing system for thermal cameras, Proc. of the 9 Advanced Infrared Technology and Applications 2007, Leon Mexico, pp. 252-262, (2008).

[7] Kastek M., Piątkowski T., Polakowski H. and Sosnowski T., Methane detection in far infrared using multispectral IR camera, 9th International Conference on Quantitative InfraRed Thermography, pp 347-350, Poland, (2008). 